\title{
Modeling of Blue Phase Liquid Crystal Displays
}

\author{
Zhibing Ge, Linghui Rao, Sebastian Gauza, and Shin-Tson Wu, Fellow, IEEE
}

\begin{abstract}
We propose a numerical model based on Kerr effect for simulating the electro-optics of polymer-stabilized blue phase liquid crystal displays (BP-LCDs). Parameters affecting the electro-optics of BP-LCDs in in-plane-switching (IPS) structures, such as wavelength, temperature, electrode dimension, and cell gap are investigated. In addition, viewing angle and color shift of IPS BP-LCDs are studied.
\end{abstract}

Index Terms-Blue phase (BP), color shift, fast response time, in-plane switching (IPS), Kerr effect.

\section{INTRODUCTION}

$\mathbf{P}$ OLYMER-STABILIZED blue phase liquid crystal displays (BP-LCDs) [1]-[5] offer several attractive features: 1) they do not require any alignment layer so that the fabrication process is simple; 2) the voltage-off state is optically isotropic so that the viewing angle is wide and symmetric; and 3) the response time is in submillisecond range (depending on the polymer concentration and the LC employed) so that it enables color sequential displays using RGB light emitting diodes [6]. However, at present, the operating voltage of BP-LCDs is still too high. There is an urgent need to develop numerical models to understand the operation mechanisms of BP-LCDs and find solutions for lowering the operating voltage.

Recently, our group has developed a crude model [5] for characterizing the electro-optical properties of BPLC materials in in-plane switching (IPS) [7] and fringe-field switching (FFS) cells [8]. However, detailed investigation on the electro-optics and display characteristics of BP-LCDs have not been systematically addressed yet.

In this paper, we first discuss the detailed formulation of our numerical model that is useful for calculating the induced birefringence of BPLCs based on the Kerr effect in IPS or FFS cell. From this model, the dependence of BPLC electro-optics on different parameters such as wavelength, temperature, electrode configuration, cell gap and LC birefringence is investigated. The viewing angle and color shift of the IPS BP-LCDs are also studied. Finally, potential approaches for reducing the driving voltage are discussed.

\section{NumericAl MOLDEING}

Typically, BPLCs exist only in a very narrow temperature range $(\sim 1-2 \mathrm{~K})$ between the isotropic and helical cholesteric

Manuscript received March 04, 2009; revised April 26, 2009. Current version published June 26, 2009. This work is supported by Chi-Mei Optoelectronics Corporation (Taiwan).

The authors are with the CREOL, The College of Optics and Photonics, University of Central Florida, Orlando, FL 32816 USA (e-mail: zge @ mail.ucf.edu; 1rao@mail.ucf.edu; sgauza@mail.ucf.edu; swu@mail.ucf.edu).

Color versions of one or more of the figures in this paper are available online at http://ieeexplore.ieee.org.

Digital Object Identifier 10.1109/JDT.2009.2022849 phase [9], [10]. According to Meiboom [10], blue phases are understood to have double twist cylinders that are arranged in a cubic lattice in different ways. The coexistence with disclination lines stabilizes such three-dimensional periodical structures. Recently, the temperature range of BPLCs is greatly expanded by the polymer stabilization method [1]-[4]. The symmetric cubic structures in polymer-stabilized BPLCs appear optically isotropic at zero electric field, but anisotropic when a strong field is applied to induce birefringence. The physical mechanism of such induced birefringence is believed to originate from the electric field-induced LC directors reorientation inside the cylinders, but the detailed physics is still not yet completely understood.

Strictly speaking, to accurately compute the detailed molecular distribution of BPLCs in response to an external field, both Landau free energy and electric energy need to be considered, which is extremely complicated [9], [10]. But macroscopically, the isotropic to anisotropic transition of BPLC can be described by the Kerr effect [11]. Thus, the electro-optical properties of a BP LCD can be simulated by computing the induced birefringence profile based on Kerr effect.

For display applications, a BPLC cell is placed between two crossed linear polarizers and horizontal electric fields generated from IPS electrodes are used to induce phase retardation for the incident light. At a null voltage state, each small blue phase unit is optically isotropic, having refractive indices identical in its principal coordinates. This leads to a very good dark state. When a strong electric field $\mathbf{E}$ is applied, birefringence will be induced and the refractive ellipsoid will have its optic axis aligned along the direction of the $\mathbf{E}$ vector (as shown in Fig. 1). The amplitude of the induced birefringence $(\Delta \mathrm{n})$ can be characterized by the following equation [5], [11]:

$$
\Delta n=\lambda K E^{2}=(\Delta n)_{\mathrm{o}}\left(E / E_{s}\right)^{2}
$$

where $\lambda$ is the wavelength, $K$ is the Kerr constant, $(\Delta n)_{\mathrm{o}}$ is the maximum induced birefringence, and the induced $\Delta n$ saturates at $(\Delta n)_{\mathrm{o}}$ when the electric field $E$ exceeds a saturation field $E_{\mathrm{s}}$. To avoid polymer network breakdown and damage to the cell, the BPLC is usually operated at $E<E_{\mathrm{s}}$.

Based on above descriptions, we propose the following flowchart to model the BP LCD as shown in Fig. 1. First, we compute the potential distribution $\Phi$ from solving the Poisson equation $\nabla(\varepsilon \nabla \cdot \Phi)=0$ and then the distribution of electric field $\mathbf{E}$ in the media. Based on the electric field, we further calculate the induced birefringence distribution $\Delta n$ from (1) and assign the local optic axis direction of each unit along the $\mathbf{E}$ vector there. According to (2), we confine the calculated birefringence from (1) to below the intrinsic birefringence $(\Delta n)_{\mathrm{o}}$ of the BPLC system. After obtaining the birefringence and optic axis distribution, we adopt the extended Jones matrix [12] to compute the related electro-optical properties, such as voltage-dependent transmittance curve (VT), viewing angle, and color shift. 


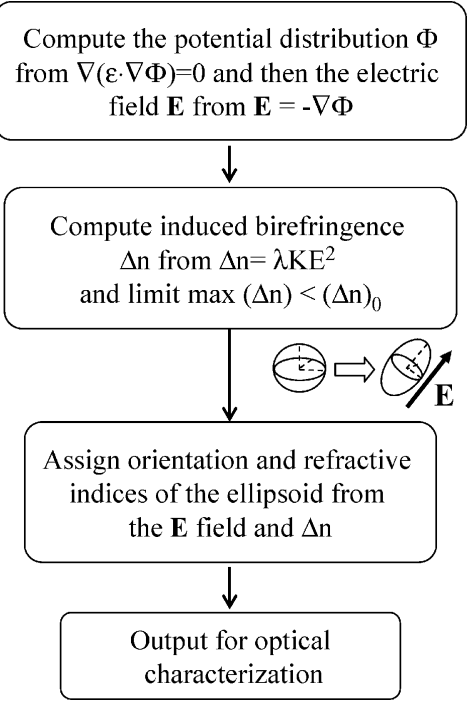

Fig. 1. Flowchart of the BP LCD modeling based on the Kerr effect (inset plot depicts the induced optical birefringence).

\section{RESULTS}

\section{A. Wavelength Effect}

The wavelength dependent LC birefringence $(\Delta n)_{\mathrm{o}}$ based $\lambda K$ can be described by the single-band model as [13], [14]

$$
\lambda K \approx G \frac{\lambda^{2} \lambda^{* 2}}{\lambda^{2}-\lambda^{* 2}} .
$$

Here $\lambda^{*}$ is the mean resonance wavelength and $\mathrm{G}$ is a proportionality constant. In an experiment, we prepared a BPLC cell in the FFS structure using a host LC material having a large dielectric anisotropy $(\Delta \varepsilon \sim 32)$ and high birefringence $(\sim 0.30)$. The monomers employed are RM-257 and EHA (ethylhexyl acrylate). From our fitting to the measured VT curve, we found the Kerr constant of the mixture is $K \sim 1.03 \times 10^{-9} \mathrm{~m} / \mathrm{V}^{2}$ at room temperature $\left(25^{\circ} \mathrm{C}\right)$ and $\lambda=632.8 \mathrm{~nm}$ [5]. The response time of the sample is $\sim 300 \mu \mathrm{s}$. For comparison, Kikuchi's group reported $K \sim 10^{-10}$ to $10^{-9} \mathrm{~m} / \mathrm{V}^{2}$ for their high $\Delta n$ and $\Delta \varepsilon$ materials [4].

In principle, from (2) we could obtain $\mathrm{G}$ and $\lambda^{*}$ values by measuring the Kerr constant of the BPLC cell at two wavelengths. To elucidate the simulation method, we just use a typical high $\Delta n$ LC mixture as an example. For E-7 type LC mixtures $(\Delta n \sim 0.22), \lambda^{*} \sim 250 \mathrm{~nm}$ due to elongated $\pi$-electron conjugation [13]. Let us assume $K \sim 1.03 \times 10^{-9} \mathrm{~m} / \mathrm{V}^{2}$ at $\lambda=632.8 \mathrm{~nm}$, thus we find $\mathrm{G} \sim 8.776 \times 10^{-3} \mathrm{~V}^{-2}$. Plugging these numbers into (2), the Kerr constant at other wavelengths can be obtained.

Fig. 2 shows the simulated VT curves of a $10-\mu \mathrm{m}$ IPS cell with electrode width $w=5 \mu \mathrm{m}$ and spacing $l=10 \mu \mathrm{m}$. The electrode strips are placed at $45^{\circ}$ with respect to the transmission axis of the top linear polarizer. The following Kerr constants are used: $K \sim 1.763 \times 10^{-9} \mathrm{~m} / \mathrm{V}^{2}$, $K \sim 1.268 \times 10^{-9} \mathrm{~m} / \mathrm{V}^{2}$, and $K \sim 0.9882 \times 10^{-9} \mathrm{~m} / \mathrm{V}^{2}$ for $\lambda=450 \mathrm{~nm}, 550 \mathrm{~nm}$, and $650 \mathrm{~nm}$, respectively. In the plot, the

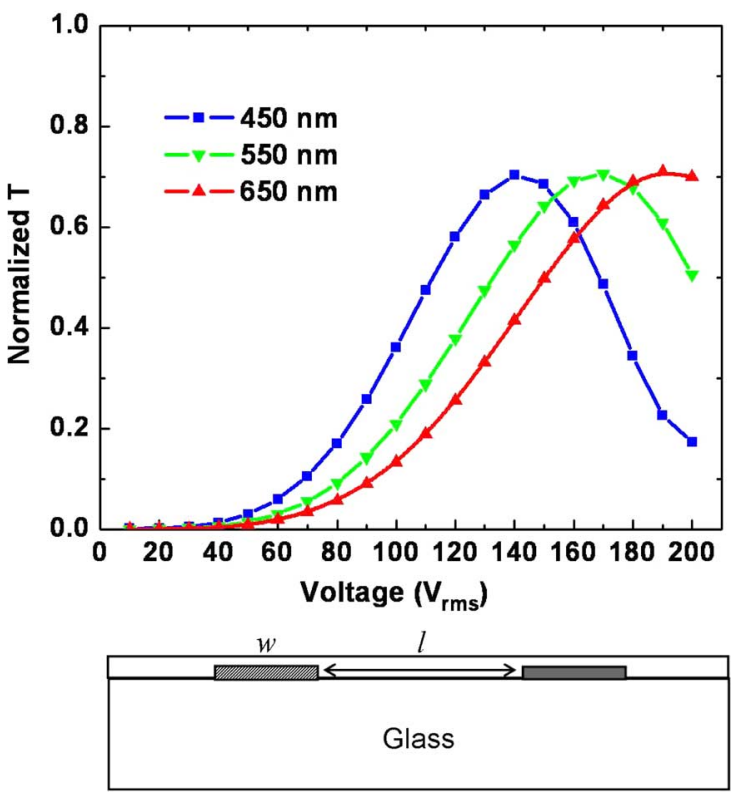

Fig. 2. VT curves of the BPLC in a 10- $\mu \mathrm{m}$ IPS cell with electrode width $w=$ $5 \mu \mathrm{m}$ and spacing $l=10 \mu \mathrm{m}$ at R $(650 \mathrm{~nm}), \mathrm{G}(550 \mathrm{~nm})$, and B $(450 \mathrm{~nm})$ wavelengths.

transmittance is normalized to the maximum value from two parallel polarizers at each wavelength.

In comparison to the nematic IPS or FFS cell, the IPS BPLC cell exhibits much larger color dispersion. In a conventional nematic IPS or FFS cell, the on-state LC profile consists of two connected TN cells with reserve twist sense [15], thus there is a self-compensation for wavelength dispersion from this special two-TN-cell profile. But in the IPS BPLC, the transmittance originates from pure phase retardation effect, similar to a vertical alignment (VA) cell. A shorter wavelength has a larger $\mathrm{K}$ constant to get a larger $\Delta n / \lambda$ value, resulting in a lower on-state voltage. However, different from the nematic VA cell, the induced birefringence (bright state) of the IPS BPLC has multi-domain structure originating from the electric field profiles. In an IPS cell, horizontal fields dominate between electrode gaps, and vertical components flourish above the electrode surfaces. Because each local induced birefringence also aligns with the electric field there, the electric-field-induced refractive ellipsoids in the whole cell will gradually align from vertically to horizontally starting from the electrode center to the electrode gap region. This multi-domain profile leads to a more symmetric and wider viewing angle, as will be discussed later.

\section{B. Temperature effect}

The temperature effect of BP LCD is based on the fact that $\lambda K$ has similar temperature dependence to birefringence. In (2), $G$ is linearly proportional to $S$, order parameter of the BPLC material. Thus we can also roughly rewrite the temperature effect as [13], [14]

$$
\lambda K \approx(\lambda K)_{\mathrm{o}} S=(\lambda K)_{\mathrm{o}}\left(1-T / T_{c}\right)^{\beta}
$$

where $T_{\mathrm{c}}$ is the clearing temperature, exponent $\beta$ is a material constant, and $(\lambda K)_{\mathrm{o}}$ is the corresponding value at $T=0 \mathrm{~K}$. In reality, during preparing the polymer-stabilized BPLC cell, 


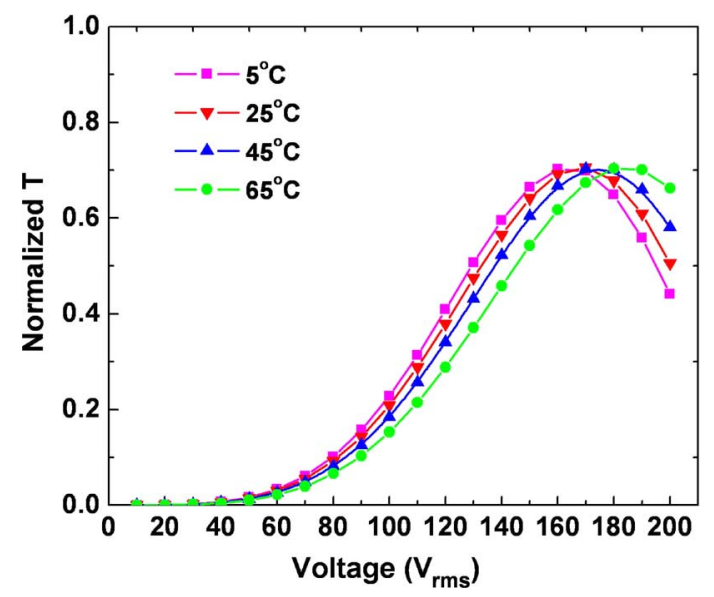

Fig. 3. Simulated VT curves of the IPS BPLC cell at $5{ }^{\circ} \mathrm{C}, 25^{\circ} \mathrm{C}, 45^{\circ} \mathrm{C}$, and $65^{\circ} \mathrm{C}$. IPS cell: $d=10 \mu \mathrm{m}, w=5 \mu \mathrm{m}$ and $l=10 \mu \mathrm{m} . \lambda=550 \mathrm{~nm}$.

once the monomers RM-257 and EHA are polymerized, they will be localized at these disclination cores in each periodic lattice at a small concentration. Thus the main bulk is still the host LC material. In other words, the temperature performance of the polymer-stabilized BPLC cell is still similar to that of the host LC material. In our experiment, the clearing temperature $T_{\mathrm{c}}$ of the host material is $96^{\circ} \mathrm{C}$. To obtain the $\lambda K$ values at different temperatures, we still have two unknowns: $(\lambda K)_{\mathrm{o}}$ and $\beta$. However, the $\beta$ value is usually between 0.20 and 0.23 for most LC materials. Here we assume $\beta \sim 0.21$ in the calculation and we also find from our calculation that $\lambda K$ is quite insensitive to $\beta$ when $\beta$ is between 0.20 and 0.23 . Based on above assumptions, we find $(\lambda K)_{\mathrm{o}} \sim 9.86 \times 10^{-16} \mathrm{~m}^{2} / \mathrm{V}^{2}$. Here one should note that while computing the VT curves at different temperatures, the temperature dependence of $(\Delta n)_{\mathrm{o}}$ needs to be taken into consideration as well. As temperature increases, both $\lambda K$ and $(\Delta n)_{\mathrm{o}}$ decrease. From Fig. 3, the VT curve of the IPS BPLC gradually shifts toward the high voltage side as the temperature increases.

\section{Electrode Effect}

As shown in (1), the induced birefringence $\Delta n$ in BPLC cell is related to $E^{2}$ and the optic axis of the new ellipsoid is along the electric field vector E. In an IPS structure, horizontal electric fields flourish between the pixel and common electrode, and vertical field dominate above the electrodes. For the BPLC cell at $45^{\circ}$ away from the polarizer's transmission axis, only the induced $\Delta n$ from horizontal electric fields contribute to the overall transmittance. Fig. 4 plots the position-dependent transmittance curves at different voltages of the IPS cell, where high transmittance occurs between the electrodes. The incident wavelength is at $550 \mathrm{~nm}$ and Kerr constant is $K \sim 1.268 \times 10^{-9} \mathrm{~m} / \mathrm{V}^{2}$. In the IPS-BPLC cell, even at a very high voltage $\left(V \sim 170 V_{\text {rms }}\right)$ the transmittance above electrodes is still negligible. This is because the optic axis of the refractive ellipsoid there is almost parallel to the incidence light. On the other hand, in a conventional nematic IPS cell, the horizontal rotation of LC molecules between the pixel and common electrodes will also produce a weak in-plane LC rotation above the electrode surfaces because the LC is a continuum material. Thus, a weak transmittance also occurs
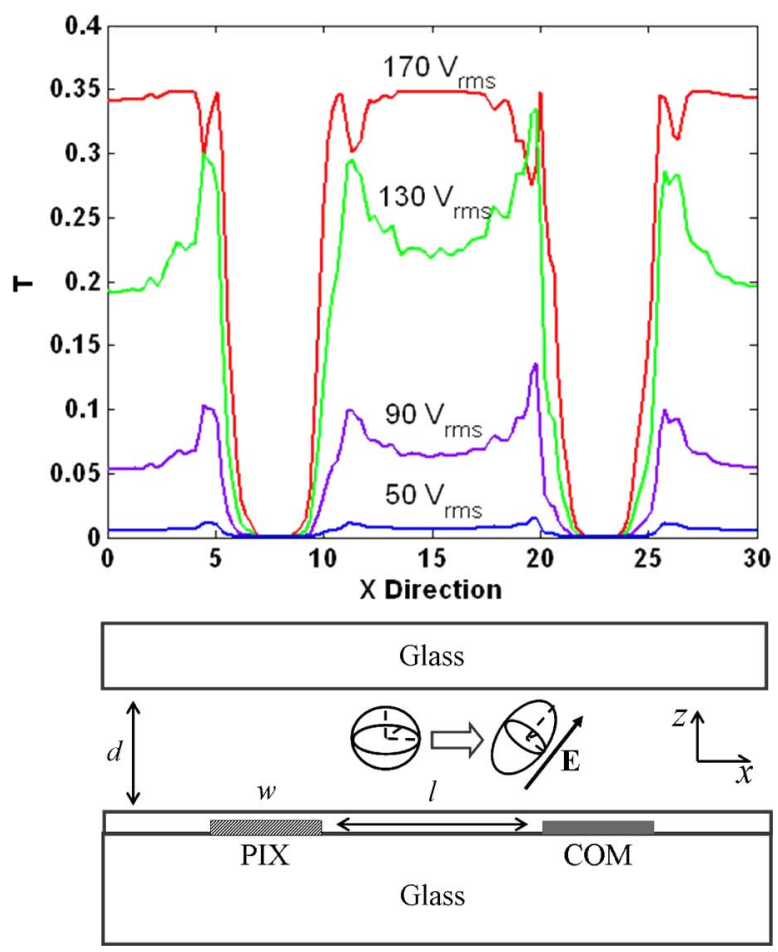

Fig. 4. Transmittance curves at different voltages of the BPLC in a $10-\mu \mathrm{m}$ IPS cell $(w=5 \mu \mathrm{m}$ and $l=10 \mu \mathrm{m})$ at $\lambda=550 \mathrm{~nm}$ (the maximum transmittance from parallel polarizers is $34.83 \%$ ).

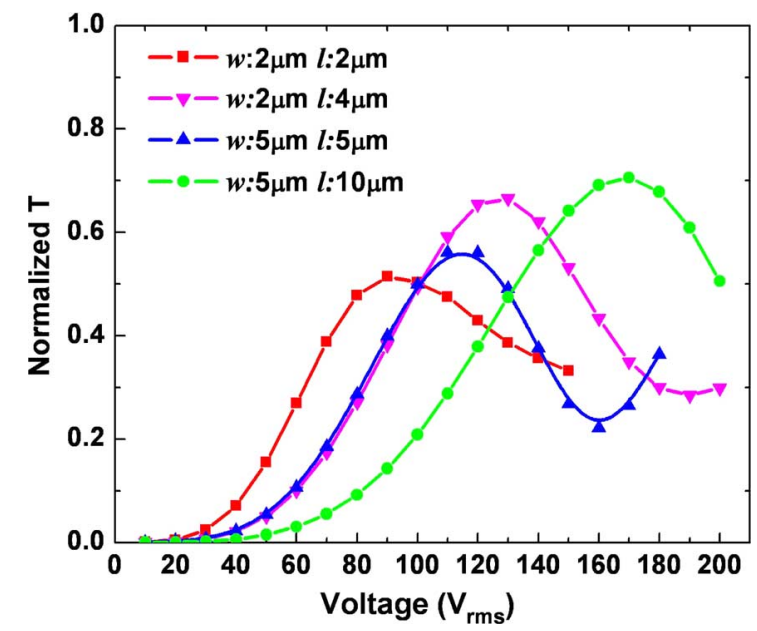

Fig. 5. VT curves of IPS-BPLC cells with different electrode dimensions at $\lambda=550 \mathrm{~nm}$

above electrodes, which enhances the overall transmittance. Nevertheless, in the IPS-BPLC cell the induced $\Delta n$ above the electrode surfaces will only act on the oblique incident light to make the viewing angle more symmetric.

The electrode dimension also plays an important role in the electro-optics of BP LCDs. Fig. 5 shows the simulated VT curves for different electrode dimensions. Generally, a smaller electrode spacing $l$ will lead to a stronger electric field intensity, which in turn results in a lower driving voltage. Since only the regions between electrodes contribute to transmittance, a larger $l / w$ ratio will bring a higher transmittance. However, the IPS cell with $w=2 \mu \mathrm{m}$ and $l=4 \mu \mathrm{m}$ exhibits a little bit higher voltage as compared to the cell with $w=5 \mu \mathrm{m}$ and $l=5 \mu \mathrm{m}$. 
In an IPS cell, the electric fields are generated from the bottom in-plane electrodes and penetrate into LC layer. The penetrating depth is proportional to $w+l$, which is typical for a Poisson problem in the form of $\nabla^{2} \phi=0$. As a result, for two IPS cells with a similar electrode spacing $l$, the one with a larger dimension $(w+l)$ will have a thicker LC layer contributing to the induced $\Delta \mathrm{n}$. As a result, the transmittance peak could occur at a lower voltage. Besides, here we can also see that a smaller $(w+l)$ also leads to a little bit lower transmittance peak, even the ratio $w / l$ is same. Because different electrode absolute dimensions have quite different penetrating depths, a same $w / l$ ratio does not necessarily lead to an identical response of transmittance to voltage. One example is the operation voltage at which overall transmittance peaks. From simulation, it is found that the transmittance at the operation voltage near electrode edges (e.g., from $x=10 \mu \mathrm{m}$ to about $12 \mu \mathrm{m}$ in Fig. 4) is a little bit higher in a cell with a larger $(w+l)$ value. Because the transmittance is averaged from whole period, the overall maximum transmittance in a larger $(w+l)$ cell also peaks a little bit higher, but not in a big difference as compared to the cells using a smaller $(w+l)$ value.

\section{Cell Gap Effect}

In a conventional nematic IPS cell, cell gap affects the transmittance and response time. However, to our surprise the cell gap effect in an IPS BPLC cell is not too obvious. Fig. 6(a) and (b) depict the induced birefringence distribution of two IPS cells with $[w=5 \mu \mathrm{m}, l=10 \mu \mathrm{m}]$ and $[w=2 \mu \mathrm{m}, l=4 \mu \mathrm{m}]$, respectively. As we can see for the first IPS cell with a larger electrode dimension, the maximum penetrating layer thickness above the electrode (defined at a vertical position where $\Delta \mathrm{n}>0.05$ ) is $\sim 3.0 \mu \mathrm{m}$, while it is only $\sim 2.0 \mu \mathrm{m}$ for the second IPS cell with a smaller dimension. In addition, from Fig. 6(a), the induced $\Delta \mathrm{n}$ between the electrode gap is below 0.1 at $170 \mathrm{~V}_{\mathrm{rms}}$. In the cell with a small electrode dimension shown in Fig. 6(b), at $\mathrm{V}=130 \mathrm{~V}_{\mathrm{rms}}$, the induced $\Delta \mathrm{n}$ reaches over 0.2 in the electrode gap, but the smaller penetrating depth in the vertical direction reduces the overall phase retardation. In both figures, although the induced $\Delta \mathrm{n}$ value peaks above the electrode surfaces, the local optic axis of the ellipsoid there is mostly vertical, thus makes no contribution to the transmittance, as verified by Fig. 4. Moreover, from both figures, we find that the penetrating depth in the vertical direction is very small from bottom electrodes regardless of the electrode dimension. In other words, the IPS BPLC cell is not critically dependent on the cell gap, as long as the cell gap is larger than the penetrating length. This is another major difference from the conventional nematic IPS cells.

Fig. 7 shows the VT curves of IPS-BPLC with two different electrode configurations and cell gaps (5 and $10 \mu \mathrm{m})$. For a given electrode configuration, the VT curve is indeed not sensitive to the cell gap variation, if cell gap is above the penetrating depth. However, for the cell with $[w=5 \mu \mathrm{m}, l=10 \mu \mathrm{m}]$, when cell gap decreases to $2 \mu \mathrm{m}$ that is below the penetrating depth, the operation voltage shifts to a higher value. This is because the accumulated phase retardation at $V=170 \mathrm{~V}_{\text {rms }}$ with induced $\Delta \mathrm{n} \sim 0.1$ between electrodes is not enough, thus a higher voltage $\left(V=190 V_{\text {rms }}\right)$ is needed to increase the induced $\Delta \mathrm{n}$ for $1 \pi$ phase retardation there in this thinner cell. On the other hand, for the IPS cell with $[w=2 \mu \mathrm{m}, l=4 \mu \mathrm{m}]$, the $2 \mu \mathrm{m}$ cell
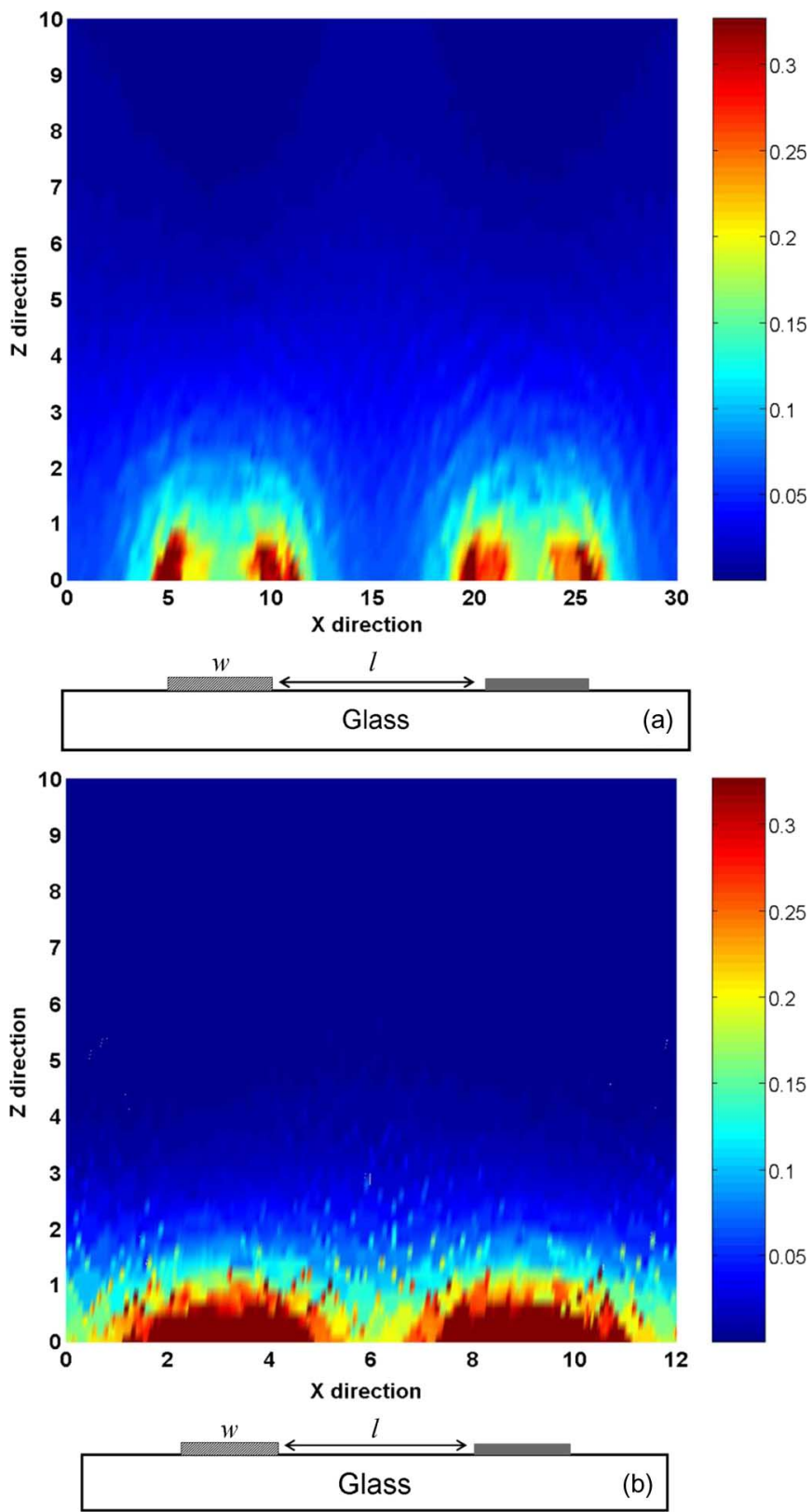

Fig. 6. Induced $\Delta \mathrm{n}$ profile of BPLC in a $10-\mu \mathrm{m}$ IPS cells at $\lambda=550 \mathrm{~nm}$ with (a) $w=5 \mu \mathrm{m}$ and $l=10 \mu \mathrm{m}$ at $170 V_{\mathrm{rms}}$ and (b) $w=2 \mu \mathrm{m}$ and $l=4 \mu \mathrm{m}$ at $130 V_{\mathrm{rms}}$

gap is still above (or close to) the penetrating depth, thus there is no significant change in attainable phase retardation at the same voltage.Here the small change in operation voltage in the curve results from the influence of the passivation layer $\mathrm{SiO}_{2}$ with dielectric constant $\sim 3.9$, which is much smaller than the LC material $(\Delta \varepsilon \sim 32)$. Thus more electric energy is attracted into the first $2 \mu \mathrm{m} \mathrm{LC}$ cell region in a thinner cell, than that in a $10-\mu \mathrm{m} \mathrm{LC}$ cell (which has $8-\mu \mathrm{m}$ LCs above the $2-\mu \mathrm{m}$ effective cell), leading to a little decrease of the voltage to reach the same transmittance.

\section{E. Birefringence Effect}

A larger intrinsic LC birefringence $(\Delta n)_{\mathrm{o}}$ not only helps to boost the Kerr constant [4] but also has direct impacts to the electro-optics in different cell dimensions. Fig. 8(a) shows the 


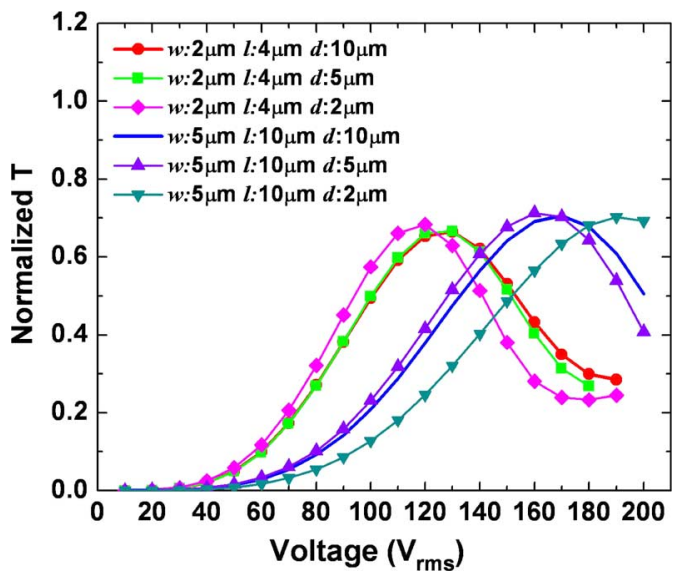

Fig. 7. VT curves of BPLC in 10- $\mu \mathrm{m}$ IPS cells with different electrode dimensions and cell gaps. $\lambda=550 \mathrm{~nm}$.

VT curves of a 10- $\mu \mathrm{m}$ IPS BPLC cell $(w=5 \mu \mathrm{m}$ and $l=$ $10 \mu \mathrm{m})$ at different $(\Delta n)_{\mathrm{o}}$ values. Referring to Fig. 6(a), at $170 V_{\mathrm{rms}}$, the region with an induced $\Delta n$ reaching over 0.30 is only confined near the electrode surfaces, where the electric fields are mostly vertical, and these regions occupy only a small portion in the horizontal direction. And the maximum induced $\Delta n$ between the electrode gap is still $\sim 0.1$. As a result, the VT curves with different $(\Delta n)_{\mathrm{o}}$ (all above 0.1) are quite close to each other. However, in the second cell with $w=2 \mu \mathrm{m}$ and spacing $l=4 \mu \mathrm{m}$, as shown in Fig. 6(b), the spacing region exhibits a larger induced $\Delta n \sim 0.2$ at $V=130 \mathrm{~V}_{\text {rms }}$. In other words, if the inherent $(\Delta n)_{\mathrm{o}}$ is smaller than 0.2 , say $(\Delta n)_{\mathrm{o}}=0.1$, it will output a smaller transmittance at $130 \mathrm{~V}_{\mathrm{rms}}$, and its maximum transmittance peak will also be lower than that using $(\Delta n)_{\mathrm{o}} \sim 0.32$ at $\lambda=550 \mathrm{~nm}$. Therefore, in IPS BPLC structure, a larger $(\Delta n)_{\mathrm{o}}$ helps to enhance the transmittance and lower the driving voltage, not only from increasing the Kerr constant (from material aspect) [5], but also from the induced $\Delta n$ distribution in the cell.

\section{F. Viewing Angle}

The BPLC behaves like an isotropic material optically when no voltage is applied in the dark state. As a result, the dark-state light leakage only occurs at an off-axis incidence that merely comes from the effective angle deviation between two crossed linear polarizers, i.e., two crossed linear polarizer at a normal incidence is no longer perpendicular to each other at an oblique incidence. On the other hand, in a voltage-on state the induced $\Delta n$ also has a multi-domain-like profile from the electric field distribution in an IPS cell. Thus, the iso-brightness contour of the IPS BPLC cell is more symmetric.

Fig. 9(a) shows the iso-contrast plot of an IPS BPLC cell at $\lambda=550 \mathrm{~nm}$, with electrode width $w=5 \mu \mathrm{m}$ and spacing $l=10 \mu \mathrm{m}$. As expected, the viewing angle is more symmetric even using an IPS structure with merely $2 \mathrm{D}$ lengthwise strips. To compensate the viewing angle, both uniaxial films and biaxial films can be employed to suppress the light leakage at dark state and expand the viewing angle [5], [16]. The contrast ratio over 200:1 can be easily expanded to over $75^{\circ}$ as shown in Fig. 9(b). The viewing angle of this simple IPS BPLCD is comparable to that of a conventional four-domain (4D) IPS structure with
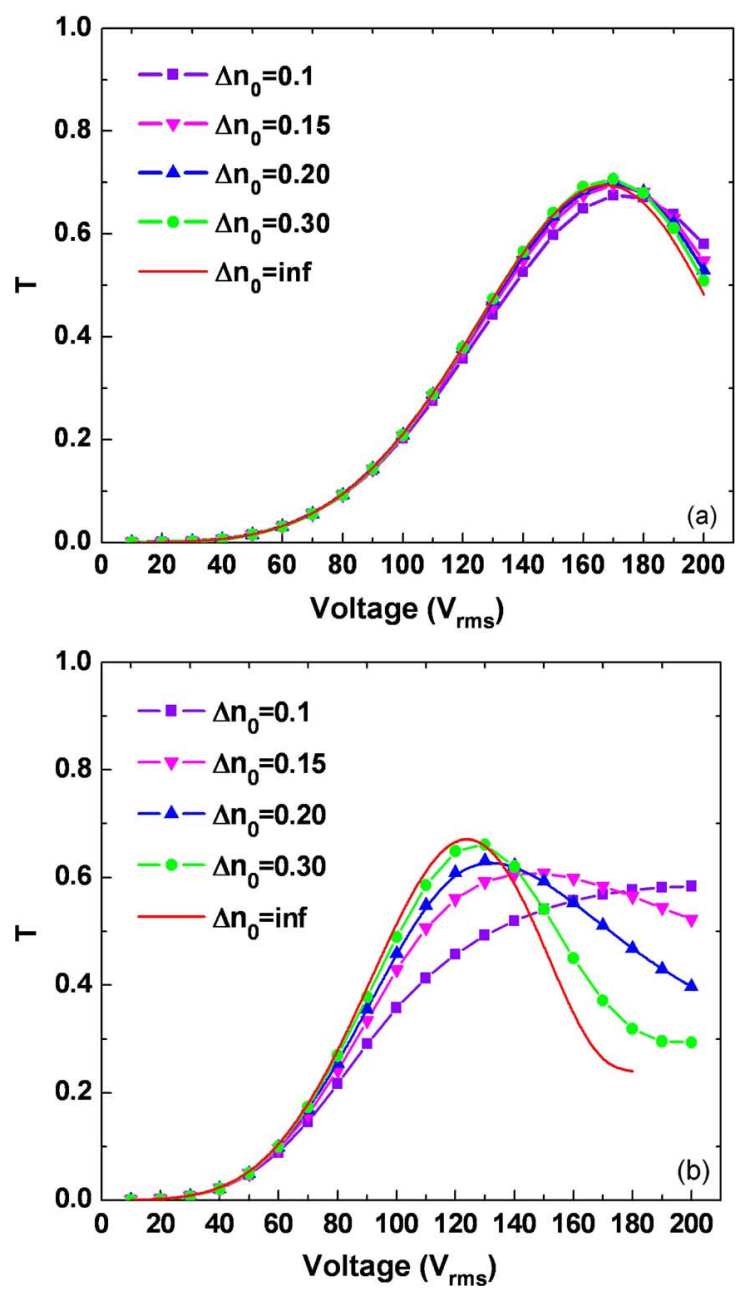

Fig. 8. VT curves of BPLC in a 10- $\mu \mathrm{m}$ IPS cells at $\lambda=550 \mathrm{~nm}$ with different inherent LC birefringence $(\Delta n)$ o having (a) $w=5 \mu \mathrm{m}$ and $l=10 \mu \mathrm{m}$, and (b) $w=2 \mu \mathrm{m}$ and $l=4 \mu \mathrm{m}$.

zigzag electrodes. Here to demonstrate the help of a multi-domain structure in expanding the viewing angle and reducing the color shift, we just utilize two orthogonal 2D BP IPS cells to mimic the 4D structure for simplicity.

\section{G. Color Shift}

Color shift is another important issue for display devices. Fig. 10(a) shows the bright state color shift in CIE 1931 of the IPS BPLC cell using a CCFL light source. The color shift is reasonably small. In the calculation, real light source data, polarizer data, compensation film data, color filter data, and the proposed $\lambda \mathrm{K}$ dispersion relation in (2) are all taken into consideration. As discussed before, the multi-domain-like distribution of induced $\Delta \mathrm{n}$ in the IPS structure with merely 2D strips helps to make the viewing angle more symmetric and reduce the color shift of the bright state as well. For color shift, if a single domain LC cell such as a uniform VA cell is adopted, the output spectrum of the visible lights will change abruptly when polar and azimuthal angle vary. When a multi-domain configuration (results from the electric field profile) is employed, wavelength dispersion at off-axis could be compensated, since the output transmittance is averaged on the whole domain and is less dependent on the polar or azimuthal angle. The color shift $\left(\Delta u^{\prime} v^{\prime}\right)$ based 

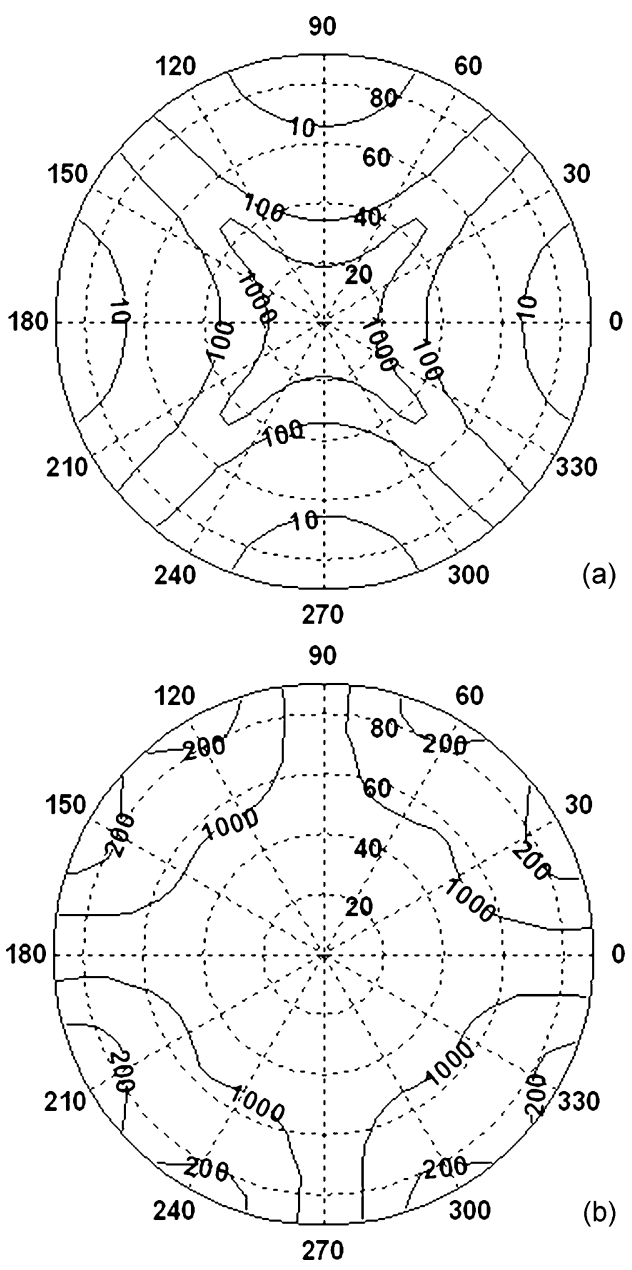

Fig. 9. Simulated iso-contrast plots of the IPS BPLC cell: (a) without any compensation film, and (b) with a biaxial film with $N_{\mathrm{z}}=0.5, \mathrm{R}_{0}=\left(n_{\mathrm{x}}-\mathrm{n}_{\mathrm{y}}\right) \cdot d=$ $\lambda / 2$. IPS cell: $d=10 \mu \mathrm{m}, w=5 \mu \mathrm{m}$, and $l=10 \mu \mathrm{m}$ and $\lambda=550 \mathrm{~nm}$.

on CIE 1976 is $~ 0.0622$ for the IPS BPLC cell using 2D strips. As mentioned above, 2D IPS BPLC cell already have certain multi-domain profile, but only in one plane (like the $x-z$ plane in Fig. 4). In a 4D structure having another orthogonal 2D structure, the output spectrum is even less dependent on the polar or azimuthal angles. The same bright state color shift for the IPS BPLC cell using $4 \mathrm{D}$ profile (here it is mimicked by two orthogonal 2D cells, but in real fabrication it can be realized by zigzag electrodes, as those widely employed in commercial IPS LCDs) are shown in Fig. 10(b), where a much smaller $\left(\Delta \mathrm{u}^{\prime} \mathrm{v}^{\prime}\right) \sim 0.026$ is obtained.

\section{H. Lower Operating Voltage}

Presently the driving voltage of the BPLC in IPS cells is still too high for practical applications. To reduce the driving voltages, a larger Kerr constant $\mathrm{K}$ is the most effective method, which closely relates to the material engineering of new host LC materials with a high dielectric anisotropy $\Delta \varepsilon$, birefringence $\Delta n$, and a large $K_{33} / K_{11}$ value [4]. Fig. 11 shows the VT curves for IPS cell using $w=2 \mu \mathrm{m}$ and $l=4 \mu \mathrm{m}$, at $K=K_{0}=1.268 \times 10^{9} \mathrm{~m} / \mathrm{V}^{2}, K=10 K_{0}$, and $K=$ $100 K_{0}$, respectively. The on-state voltage drops from $130 V_{\text {rms }}$ at $K=\mathrm{K}_{0}$ to $\sim 40 V_{\mathrm{rms}}$ by $\sim 3.2 \mathrm{X}$ at $K=10 K_{0}$, and to
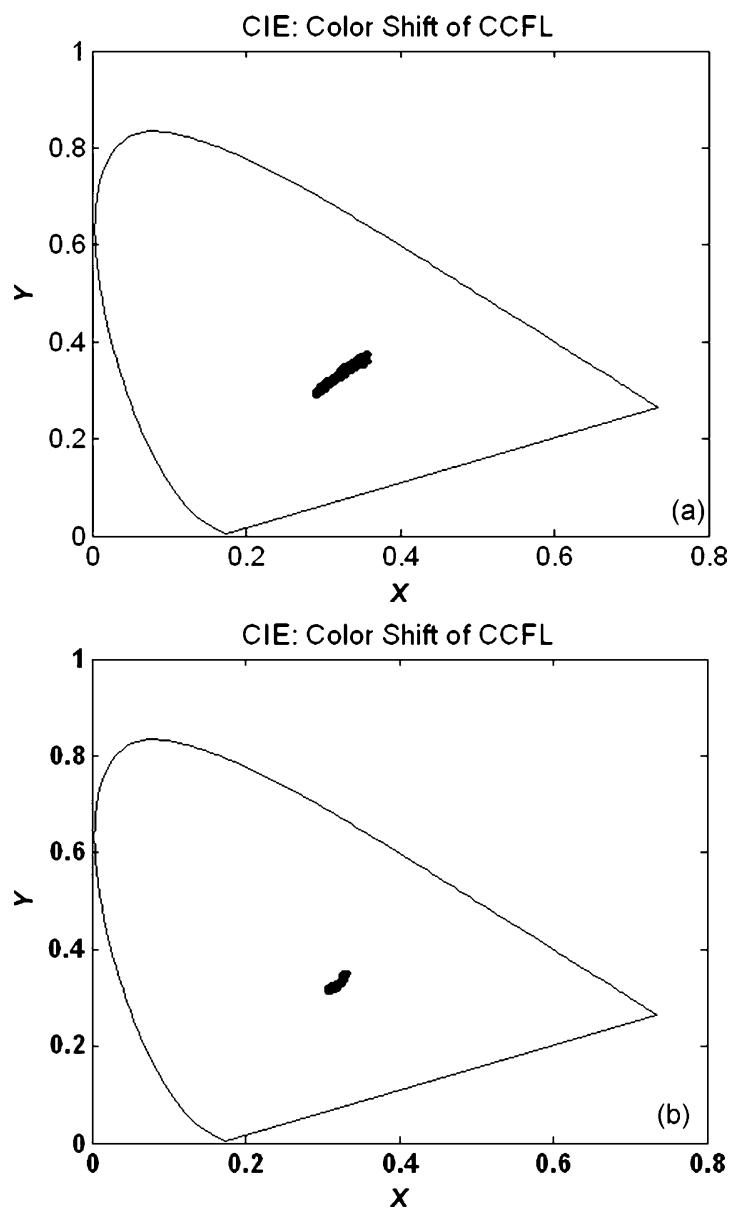

Fig. 10. Simulated bright state color-shift of the IPS BPLC cell: (a) with 2D strips and (b) with 4D structure (by assuming two orthogonal 2D structures) Here IPS cell parameters are: $\mathrm{d}=10 \mu \mathrm{m}, w=5 \mu \mathrm{m}$ and $l=10 \mu \mathrm{m}$ and no compensation film is used.

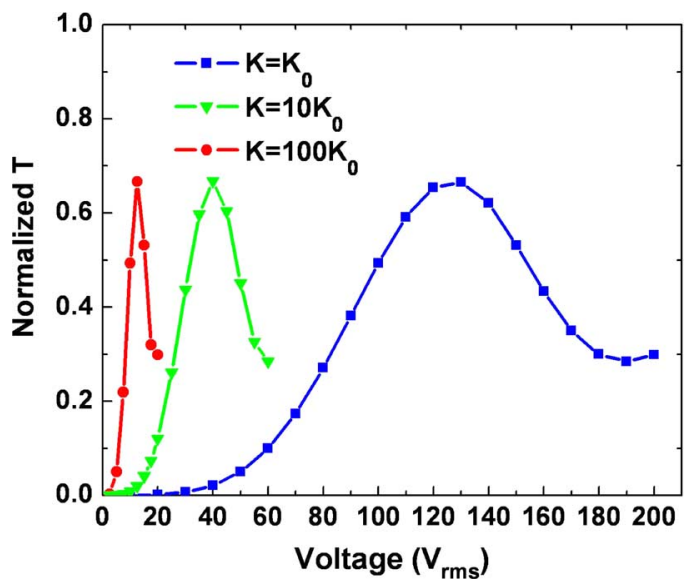

Fig. 11. Simulated VT curves for the IPS BPLC cell with $d=10 \mu \mathrm{m}, w=$ $2 \mu \mathrm{m}$, and $l=4 \mu \mathrm{m}$ and $\lambda=550 \mathrm{~nm}$ with $K=\mathrm{K}_{0}=1.268 \times 10^{-9} \mathrm{~m} / \mathrm{V}^{2}$, $K=10 K_{0}$, and $K=100 K_{0}$.

$\sim 12.5 V_{\text {rms }}$ by $\sim 10 \mathrm{X}$ at $K=100 K_{0}$. According to (1), we can estimate the operating voltage from the following relation $V_{1} / V_{2} \approx \sqrt{K_{2} / K_{1}}$. Besides $\Delta n$ and $\Delta \varepsilon$, monomer concentration also affects the Kerr constant. A proper monomer concentration that can stabilize the blue phases at a weakest polymer 
network will also help to reduce the driving voltage [17]. However, the tradeoff is in the slower response time. From our experimental observations, the slower response time might originate from the following factor. If a weak network cannot or cannot strongly stabilize the BP, the whole mixture might actually be in a state that is between a pure cholesteric (or nematic) phase and a pure BP. And when a high voltage is applied or released, the interaction of LC molecules will not be confined in each single small unit domain as in a pure polymer-stabilized BP LC cell. Such interaction among molecules in a larger scale slows the response time in a similar way as a nematic LC cell, where the thick cell gap employed in samples start to play a critical role.

\section{CONCLUSION}

We propose a numerical model that can characterize the Kerr effect in polymer-stabilized BP-LCDs. Based on this model, different effects, such as wavelength, temperature, electrode dimension, and cell gap, on the electro-optics of IPS BPLC cells are discussed at a more insightful way. In addition, we also find the origins for symmetric viewing angle and weak color shift of IPS BPLC structures even using a 2D electrode structure. We believe this model provides a useful tool to understand the underlying physics and optics of BP LCDs, and also to improve the performance by optimizing the display structures.

\section{REFERENCES}

[1] H. Kikuchi, M. Yokota, Y. Hiskado, H. Yang, and T. Kajiyama, "Polymer-stabilized liquid crystal blue phases," Nat. Mater., vol. 1, pp. 64-68, 2002.

[2] Y. Haseba, H. Kikuchi, T. Nagamura, and T. Kajiyama, "Large electrooptic Kerr effect in nanostructured chiral liquid-crystal composites over a wide temperature range," Adv. Mater., vol. 17, p. 2311, 2005.

[3] Y. Hisakado, H. Kikuchi, T. Nagamura, and T. Kajiyama, "Large electro-optic Kerr effect in polymer-stabilized liquid-crystalline blue phases," Adv. Mater., vol. 17, p. 96, 2005.

[4] S.-W. Choi, S.-I. Yamamoto, Y. Haseba, H. Higuchi, and H. Kikuchi, "Optically isotropic-nanostructured liquid crystal composite with high Kerr constant," Appl. Phys. Lett., vol. 92, p. 043119, 2008.

[5] Z. Ge, S. Gauza, M. Jiao, H. Xianyu, and S. T. Wu, "Electro-optics of polymer-stabilized blue phase liquid crystal displays," Appl. Phys. Lett., vol. 94, p. 101104, 2009.

[6] J. H. Lee, X. Zhu, and S. T. Wu, "Novel color-sequential transflective liquid crystal displays," J. Display Technol., vol. 3, no. 1, pp. 2-8, Mar. 2007.

[7] M. Ohe and K. Kondo, "Electro-optical characteristics and switching behavior of the in-plane switching mode," Appl. Phys. Lett., vol. 67, pp. 3895-3897, 1995.

[8] S. H. Lee, S. L. Lee, and H. Y. Kim, "Electro-optic characteristics and switching principle of a nematic liquid crystal cell controlled by fringefield switching," Appl. Phys. Lett., vol. 73, pp. 2881-2283, 1998.

[9] P. G. de Gennes and J. Prost, The Physics of Liquid Crystals, 2nd ed. Oxford: Clarendon, 1993.

[10] S. Meiboom, J. P. Sethna, W. P. Anderson, and W. F. Brinkman, "Theory of the blue phase cholesteric liquid crystals," Phys. Rev. Lett., vol. 46, pp. 1216-1219, 1981.

[11] A. Yariv and P. Yeh, Optical Waves in Crystal: Propagation and Control of Laser Retardation. Hoboken, NJ: Wiley, 2002.
[12] Z. Ge, T. X. Wu, X. Zhu, and S. T. Wu, "Reflective liquid crystal displays with asymmetric incidence and exit angles," J. Opt. Soc. Amer. A., vol. 22, pp. 966-977, 2005.

[13] S. T. Wu, "Birefringence dispersions of liquid crystals," Phys. Rev. A., vol. 33, pp. 1270-1274, 1986.

[14] S. T. Wu, C. S. Wu, M. Warenghem, and M. Ismaili, "Refractive index dispersions of liquid crystals," Opt. Eng., vol. 32, pp. 1775-1780, 1993.

[15] Z. Ge, S. T. Wu, S. S. Kim, J. W. Park, and S. H. Lee, "Thin cell fringefield-switching liquid crystal display with a chiral dopant," Appl. Phys. Lett., vol. 92, p. 181109, 2008.

[16] X. Zhu, Z. Ge, and S. T. Wu, "Analytical solutions for uniaxial-filmcompensated wide-view liquid crystal displays," J. Display Technol., vol. 2, no. 1, pp. 2-20, Mar. 2006.

[17] Y. H. Fan, Y. H. Lin, H. Ren, S. Gauza, and S. T. Wu, "Fast-response time and scattering-free polymer network liquid crystals for infrared light modulators," Appl. Phys. Lett., vol. 84, pp. 1233-1235, 2004.

Zhibing Ge received the B.S. degree in electrical engineering from Zhejiang University, Hangzhou, China, in 2002, and the M.S. and Ph.D. degrees in electrical engineering from University of Central Florida (UCF), Orlando, in 2004 and 2007 , respectively.

Since 2008, he has been with the College of Optics and Photonics at University of Central Florida, Orlando, as a research scientist. His research interests include novel liquid crystal displays and laser beam steering technologies. He has published 1 book chapter, over 30 journal papers, and 12 issued or pending patents in related area.

Dr. Ge is a recipient of the 2008 Otto Lehmann Award. Since May 2008, he has served as an associate editor for Journal of Society for Information Display (JSID) on the LCD division.

Linghui Rao received the B.S. degree in Optics from Huazhong University of Science and Technology in 2007. She is currently working toward the Ph.D. degree in the College of Optics and Photonics, University of Central Florida. Her current research interests include novel LC materials for advanced LCD applications, and blue phase liquid crystal displays.

Sebastian Gauza received the Ph.D. degree in chemistry from the Military University of Technology, Warsaw, Poland, in 2001. He is currently a research scientist at College of Optics and Photonics, University of Central Florida. His current research is to develop novel high birefringence nematic liquid crystals, blue phase liquid crystals for display and photonic applications. He has published over 50 journal papers and several book chapters.

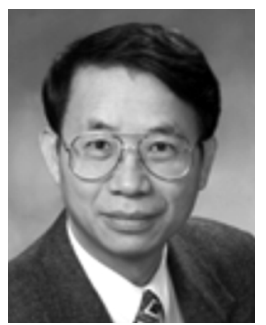

Shin-Tson Wu (M'98-SM'99-F'04) received the B.S. degree in physics from National Taiwan University, and Ph.D. degree from the University of Southern California, Los Angeles.

$\mathrm{He}$ is a PREP professor at College of Optics and Photonics, University of Central Florida (UCF). Prior to joining UCF in 2001, he worked at Hughes Research Laboratories, Malibu, CA, for 18 years. He has co-authored 5 books, 6 book chapters, over 300 journal publications, and more than 58 issued patents.

Dr. Wu is a recipient of SPIE G. G. Stokes award and SID Jan Rajchman prize. He was the founding Editor-in-Chief of IEEE/OSA JOURNAL OF DISPLAY TECHNOLOGY. He is a Fellow of the Society of Information Display (SID), Optical Society of America (OSA), and SPIE. 